myopathy (IMNM,7)) and 30 age-/sex-matched HC (25 females/5 males, mean age 54.9) without rheumatic/tumor diseases or manifest cardiovascular event were included. PM/DM patients fulfilled Bohan/Peter criteria for PM/DM. Anthropometric parameters and body composition were assessed (by densitometry-iDXA Lunar, and by bioelectric impedance-BIA2000-M), and physical activity was evaluated using Human Activity Profile (HAP) questionnaire. Routine biochemistry analysis was performed after 8 hours of fasting. Muscle involvement was evaluated by manual muscle test (MMT)-8. Data are presented as mean \pm SD.

Results: Compared to $\mathrm{HC}$, patients with IIM had significantly increased body fat $\%$ as assessed by iDXA (BF\%: $38.7 \pm 6.7$ vs. $42.5 \pm 7.1 \%, p=0.015)$, but decreased lean body mass as assessed both by iDXA (LBM: $45.7 \pm 6.6$ vs. $40.3 \pm 7.0 \mathrm{~kg}$, $\mathrm{p}=0.0005$ ) and BIA (LBM: $53.2 \pm 8.5$ vs. $48.7 \pm 9.0 \mathrm{~kg}, \mathrm{p}=0.0295$ ), and increased $\mathrm{ECM} / \mathrm{BCM}$ ratio (extracellular mass/body cell mass: $1.00 \pm 0.12$ vs. $1.43 \pm 0.42$, $p<0.0001$ ), which reflects worse muscle predispositions for physical exercise, aerobic fitness/performance, and also increases with deteriorating nutritional status. Compared to HC, IIM patients had significantly lower bone mineral density (BMD: $1.16 \pm 0.10$ vs. $1.05 \pm 0.11 \mathrm{~g} / \mathrm{cm}^{2}, p=0.0010$ ), and were currently able to perform less energetically demanding physical activities according to HAP score $(86.3 \pm 5.9$ vs. $49.0 \pm 20.2, p<0.0001)$. Disease duration negatively correlated with BMD $(r=-0.392, p=0.004)$ and LBM-BIA $(r=-0.272, p=0.047)$. CRP was positively associated with BF\% assessed both by DEXA ( $r=0.276, p=0.035)$ and $\mathrm{BIA}(\mathrm{r}=0.306, \mathrm{p}=0.025)$. MMT-8 score negatively correlated with $\mathrm{ECM} / \mathrm{BCM}$ ratio $(r=-0.385, p=0.006)$, and physical activity (HAP) negatively correlated with $\mathrm{BF} \%>\mathrm{DEXA}(\mathrm{r}=-0.292, \mathrm{p}=0.032)$.

Conclusions: Compared to healthy age-/sex-matched individuals we found significant negative changes in body composition of our IIM patients, which are associated with their disease duration, inflammatory status, skeletal muscle involvement, and physical activity, and could reflect their impaired nutritional status and predispositions for physical exercise, aerobic fitness and performance.

Acknowledgements: Supported by AZV-16-33574A, GAUK-214615.

Disclosure of Interest: None declared

DOI: 10.1136/annrheumdis-2017-eular.4824

\section{AB0672 RISK FACTORS AND TREATMENT OF RECURRENT DERMATOMYOSITIS AND POLYMYOSITIS}

S. Yamada, H. Yamashita, Y. Takahashi, H. Kaneko. Division of Rheumatic Diseases, National Center for Global Health and Medicine, Tokyo, Japan

Background: Patients with polymyositis (PM) and dermatomyositis (DM) often have repeated exacerbations of myositis. Sometimes immunosuppressive agents are added to induce remission in steroid-resistant cases, with steroid reduction in maintenance therapy, and to prevent exacerbations. However, comparative clinical studies are difficult to conduct and the appropriate immunosuppressive agents are not known [1-2]. There are also no studies of the treatment in recurrent cases with repeated relapses.

Objectives: In this study, we defined myositis that relapses more than once as recurrent myositis and examined its risk factors and re-induction treatments.

Methods: Patients from our PM and DM database who were hospitalised between January 1991 and September 2016 were reviewed. We included only patients who had been followed for more than 1 year.

Results: There were 14 cases $(13.1 \%)$ with recurrent myositis. The average observation period was 11.3 and 8.0 years in the relapsing and non-relapsing groups, respectively $(P=0.18)$, and the average ages at onset were 46.2 and 55.0 years $(P=0.17)$. The myositis relapse rate was significantly higher in the anti-aminoacyl-tRNA synthetase (ARS) antibody-positive group than in the group with other antibodies [8/24 (33.3\%) vs. $0 / 14(0.0 \%)$ cases; $P=0.02]$. The myositis relapse rate was significantly lower in the group in which one more immunosuppressive agent was combined in the initial treatment than in the group without any $[3 / 53(5.7 \%)$ vs. $11 / 48(22.9 \%)$ cases; $P=0.02]$. The myositis relapse rate did not differ significantly between DM and PM, men and women, the groups with or without malignancy, the groups with or without interstitial pneumonia (IP), and groups with or without the following initial findings: fever, joint pain, dysphagia, constipation, bedridden, muscle pain, distal muscle weakness, cutaneous symptoms (e.g., heliotrope rash, Gottron's sign, V signs, Raynaud's phenomenon, skin ulcers and necrosis, and itching sensation), cardiac complications, hypertension, diabetes, and smoking history. There was no significant difference in the serum creatine kinase and C-reactive protein between the relapse and no-relapse groups. Only 3 of 14 cases $(21.4 \%)$ had successful re-remission induced and were on maintenance therapy. In two of these cases, two immunosuppressive agents other than glucocorticoid were added: tacrolimus and methotrexate in one and tacrolimus and mycophenolate mofetil in the other. The other 11 patients were treatment-resistant.

Conclusions: The presence of anti-ARS-antibody and initial treatment with glucocorticoid only (without any other immunosuppressive agent) were the only two risk factors for recurrent myositis; none of the other factors examined were significant. Recurrent myositis is often treatment-resistant; if one immunosuppressive agent cannot introduce remission, it is worth trying two agents.

References:

[1] Gordon PA, et al: Immunosuppressant of relapses and immunomodulatory treatment for dermatomyositis and polymyositis. Cochrane Database Syst Rev 2012; 15(8):CD003643.

[2] Dalakas MC, et al: A controlled trial of high-dose intravenous immune globulin infusions as treatment for dermatomyositis. N Engl J Med. $1993 \mathrm{Dec}$ 30;329(27):1993-2000.

Acknowledgements:.

Disclosure of Interest: None declared

DOI: 10.1136/annrheumdis-2017-eular.2529

\section{AB0673 TESTING FOR ANTIBODIES SPECIFIC TO FIBRILLARIN (U3 RNP) SHOWS ADDED VALUE FOR DIAGNOSIS OF SYSTEMIC SCLEROSIS}

I. Gehring ${ }^{1}$, S. Sjölander ${ }^{2}$, P. Höpfl ${ }^{1}$, S. Swiniarski ${ }^{1}$, L. Vinderslev Iversen ${ }^{3}$, G. Gallo ${ }^{4}$. ${ }^{1}$ Thermo Fisher Scientific, Freiburg, Germany; ${ }^{2}$ Thermo Fisher Scientific, Uppsala, Sweden; ${ }^{3}$ Department of Dermatology, Aarhus University Hospital, Aarhus, Denmark; ${ }^{4}$ Thermo Fisher Scientific, Milano, Italy

Background: Systemic sclerosis (SSc) is a heterogeneous and often systemic autoimmune disorder characterised by specific autoantibody subsets. The disease is associated with anti-nuclear antibodies (ANA) and the detection of each of the autoantibodies is useful during diagnosis. Most SSc patients are positive for anti-centromere or anti-Scl70 antibodies. The presence of more rare antibodies to, e.g. Fibrillarin (U3 RNP), RNA-polymerase III and PM-Scl are often indicative of a systemic or a limited skin involvement.

Objectives: In this in-house study the prevalence, and clinical sensitivity and /specificity of anti-Fibrillarin antibodies in a subset of patients diagnosed with SSc was investigated. The major aim was to find out whether Fibrillarin testing would identify patients which are negative for standard parameters and therefore add value forin the diagnosticis workup for patients with suspected SSc.

Methods: Sera from 187 SSc patients, 53 blood donors and 177 disease controls were analysed for the presence of antibodies to CENP, Scl-70s, PM-Scl, RNA Pol III and Fibrillarin using the EliA platform (Thermo Fisher Scientific, Freiburg, Germany).

Results: In 149 out of the 187 patients one or more of the tested parameters were detected. Fibrillarin was identified in 7 out of the 149 patients including 6 solely positive for Fibrillarin. Overall sensitivity within this study is $79,68 \%$ with an overall specificity of $92,17 \%$; for Fibrillarin sensitivity of $3,74 \%$ and specificity of $98,70 \%$ was shown within this cohort.

Conclusions: Increased diagnostic sensitivity was shown in this cohort of 187 patients with SSc by adding the analysis of antibodies against Fibrillarin. Testing allowed additional identification of 6 SSc patients which correlates to an increase of $3,74 \%$ in sensitivity.

\section{References:}

[1] Arthritis Res Ther 2003, 5:80-93.

[2] Rheumatology 2001;40:1157-1162.

Disclosure of Interest: None declared

DOI: 10.1136/annrheumdis-2017-eular.4685

\section{AB0674 YKL-40 IS THE BIOMARKER FOR THE PRESENCE OF INTERSTITIAL PNEUMONIA AND PULMONARY ARTERIAL HYPERTENSION IN SYSTEMIC SCLEROSIS}

T. Furukawa, K. Matsui, M. Kitano, Y. Yokoyama, M. Sekiguti, N. Azuma, $\mathrm{H}$. Sano. Division of Rheumatology, Department of Internal Medicine, Hyogo College of Medicine, Nishinomiya, Japan

Background: SSc is a refractory of connective tissue disease that causes fibrosis of the skin and various organs. Recently, it is attention to 2013 ACR/EULAR classification criteria because can diagnose early phase before causing skin involvement in SSc. Thus, we considered that YKL-40 which is known to be involved in inflammation, tissue fibrosis and remodeling, is useful marker for evaluating early diagnosis and complications in SSc. YKL-40 is a chitinase-like protein and expressed in the synovial membrane and cartilage fibroblast cells in Rheumatoid Arthritis. Several cases of relationship between SSc and YKL-40 have been reported overseas, but it is unclear. Our report is the first in Japan.

Objectives: We investigated serum YKL-40 levels and examined immunohistochemistry (IHC) with YKL-40 of cutaneous tissue in SSc patients. To clarify YKL-40 is useful biomarker in early diagnosis in the presence or absence of complications in Japanese SSc patients.

Methods: Between August 2014 and March 2016, we treated 57 SSc patients in our department. We excluded infection, malignant disease, and other active complication may be a factor that increases YKL-40 levels. The patients were divided into 4 groups depending on whether suffered IP or PAH, which can affect the prognosis. Group1 $(n=30)$ did not suffer from either IP or PAH, Group2 $(n=12)$ suffered from IP, Group3 ( $n=7)$ suffered from PAH, and Group4 $(n=8)$ suffered from both IP and PAH. YKL-40 levels in 4 groups and a control group of healthy individuals $(n=13)$ were measured by ELISA. And age percentile strata of YKL-40 were calculated because serum YKL-40 levels have reported to rise with age; YKL-40 age percentile $=100 /\left(1+\left(\right.\right.$ Serum YKL-40 levels $\left.\left.{ }^{-3}\right) \times\left(1.062^{\text {age }}\right) \times 5000\right)$. $\mathrm{IHC}$ with YKL-40 of skin biopsy tissue from patients and controls was performed to investigate $\mathrm{YKL}-40$ expression.

Results: YKL-40 age percentile were elevated in Group1 compared to controls $(42.0 \pm 24.5$ vs $21.8 \pm 15.6)$, and were elevated due to complications (Group2, 3 , 4: $61.8 \pm 21.4,88.9 \pm 8.2,94.4 \pm 8.7$ ), with a tendency for PAH to cause greater elevation. There was no significant difference between Group3 and Group4, and 